at all about it, drugs do good to patients with pneumonia. The paper has shown the results of the experience of the writer in support of the value of drugs in the treatment of pneumonia. The remarks of the last speaker show that while preumonia is undoubtedly due to the diplococcus, yet many cases are the result of mixed infection. It is impossible, therefore, to lay down any hard and fast rules for the treatment of cases of acute pneumonia, yet there are several principles which can be followed by the practitioner and will aid him in the treatment of these cases. In the portion of the country to which the speaker belongs, pneumonia produces the most numerous fatalities; the number of cases treated each year is very large. The experience he has had in his last 200 cases of croupous pneumonia has led him to be positive--if he could be positive about anything-- that the condition can be relieved by blood-letting. There are two conditions to be overcome: 1 , the condition of the blood, and 2 , the wealness of the heart which follows. Either you must relieve the stasis in the blood-vessels or increase the power behind to force the blood through the constricted blood-ressels. Among many practitioner's veratrum viride has been lauded as almost a specific in the treatment of the first stage of pneumonia. Others rely on aconite for its dilating action on the blood-vessels, thus reliev. ing the strain on the heart. He has abundant testimony that by the use of veratrum viride the course of the disease is cut short and the case convalesces two days earlier, and the whole course is milder than where this is not given. His own preference, however, is for aconite, which acts in the same manner. By following out this treatment he has seldom had a case last longer than five days before the crisis occurred. The next point in the therapy is to strengthen the heart so that, after the action of the veratrum viride or aconite has overcome the constriction of the vessels, the blood may be forced through them. Digitalis and especially digitoxin have been recommended for their physiologic action on the heart, but in strychnin we have a remedy, which is most useful in this condition. If there is any disease in the world in which we can lecognize the efficacy of blood therapeutics, it is in acute pneumonia. In some parts of the country the mortality reported from hospitals is 25 per cent. In his portion of the country, if he did have any mortality at all, he would lose his practice, as the people expect pneumonia cases to get well if they receive proper attention.

Dr. Boyce-An important question in connection with the subject under discussion is the value of serumtherapy. To the speaker acute pneumonia is the type of a toxic disease. It has its nearest analogue among infectious diseases in diphtheria. The diplococcus of pneumonia and the bacillus diphtherix, or Klebs-Loeffler bacillus, are the sole micro-organisms which cause fibrous exudation upon mucous membranes. The clinical type of pneumonia depends entirely on the virulence of the bacillus and absorption of toxins. The clinical course of the disease is evidence of this. You may see your patient on the sixth day and he is a sick man; apparently he is still as sick as on any day previous; he has an exudate in the lung that is the same as the previous day, and suffers from fever and other evidiences of this disease, but you go back again on the same afternoon and find the patient a well man; the oppression is gone, the fever has gone and he feels well, and you can see that he is relieved, but the exudate has not changed. No diamostician can show any change in the exudate, but there is no dyspnea and the patient feels well and is hungry. The change that has taken place in the symptoms is very evident, but it has not been due to any change in the condition of the lung. On the other hand, we will have very great damage to the lung without the symptoms of the kind we see in pneumonia. For instance, we may have one of those large pleural exudates which come upon us and force the lung and heart to one side and leave only a portion of one lung for the patient to breathe with, and yet the patient will not have the dyspnea that he has in pneumonia. Therefore, the condition is not mechanical, but is due to the presence of certain toxins in the blood. Therefore, the best thing we can do for pneumonia patients is to dilute the toxinemic condition of the blood by saline injections, using the normal salt solution for this purpose; also local applications to the chest, ice-bags or hot poultices, the object being to relieve the nerves and to relieve pain, and give symptomatic relief, and by this means we do give relief to the symptoms. The treatment of serumtherapy is possible. As to the administration of veratrum viride, we know that the exudate can not be absorbed hy this agent. I have seen cases of acute pneumonia in which the physical signs all disappeared in one day, and this occurred just as often in those who had taken veratrum viride as in those who had not. As regards the comparison instituted by Dr. Melvin between cases treated br blood-letting and those who are not, taking the violence of the attack into consideration and the difference between the patients treated in private practice and those in the hospital, 25 per cent. mortality is practically correct. He knew of a hospital which for a few years had lost over 40 per cent. in acute pneumonia, simply owing to the character of the clinical material. He thought that 25 per cent. in hospital practico corresponded to a mortality of 10 per cent. in private prac. tice.

Dr. E. W. Mrtenefl, Cincinnati, Ohio, fully believes in toxins from the pneumococcus. From his observation of the disease in the hospital he has come to hold the view that acut. pneumonia is an infection and not an inflammation; that the essential factor in the pathology is not the mechanical one. but the toxin effects. We hope for the development of a better treatment with the aid of antitoxin. If the analogy is so close between diphtheria and pneumonia we should hop before long to have the antitoxin which shall be as efficacious. as the diphtheria antitoxin. The comparison has already been made between the class of patients which come into the public hospitals, the old drunkards, with liver and kidneys in a degenerate condition, the heart fatty, and whom no drugs will b. able to save, and private patients. It is remarkable that deat], statistics do not show much difference between the death-rate of pneumonia-which had a death-rate of 40 per cent. fifty. years ago-and that of the present day with all the advances of modern therapeutics. About our only hope in therapeutics. therefore, appears to be in the serum treatment. According to the last speaker, the normal salt solution is of value in acuto pneumonia, and this seems reasonable. The speaker has not much faith in any form of local application, but his own prefer. ence has been for the hot bath, and internally he uses digitalin and strychnin. He is not sure, however, that he has obtained as favorable results as Dr. Eichberg claimed in his paper. Our resident physicians in the hospital are acute observers. A few days ago, at his hospital, the residents reported that they had noticed that all the cases treated by the hot bath had died. while those who did not have the baths got well. In private practice he has obtained the most satisfactory results from the hot bath. He has also had very good results from the use of oxygen in pneumonia. He regards it as a valuable heart stimulant. The most valuable part of the treatment is fresh air, and he said that he would take out the side of the houso or drag the patient's bed into the open air if possible or necessary.

\section{IRRIGATION OF THE COLON AS A THERAPEUTIC MEASURE.*}

GEO. J. LOCHBOEHIER, PH.D., M.D.

Assistant Professor of Materia Medica and Therapeutics and Le turer on Pharmacy, School of Medicine, Georgetown University. WASHINGTON, D. C.

The introduction of water into the bowel per anum is made by injection and irrigation. In the former, only a small amount of fluid is required; in the latter, the quantity is indefinite, as it is immediately allowed to again leave the system. The fluid acts in part mechanically as a foreign substance, provokes expulsion movements, as a solvent for intestinal catarrhal products, and, where large quantities are used, drags upon the upper intestinal coils, causing thermal changes and peristalsis.

These large quantities are introduced by means of the rectal tube, made of soft rubber, preferably with the opening at the end, to prevent clogging by fecal masses, as often occurs where the tube opens on the side. An ordinary fountain syringe is attached to the tube and the flow and pressure of the fluid is readily controlled by constricting the tubing. It sometimes happens that the tube coils upon itself when it enters the sigmoid flexure and the liquid is prevented from passing farther into the bowel. 'Turck, ${ }^{1}$ of Chicage, has devised a special form of tube to obviate this. From two to four quarts of water are required, the temperature of which varie: according to the nature of the case in hand.

* Presented to the Section on Materia Medica, Therapeutics and Pharmacy at the Fifty-first Annual Meeting of the American Medical Association. held at Atlantic City, N. J., June 5-8, 1900. 
As these irrigations act mechanically in that the weight of the fluid draws upon the upper portion of the intestines, they are often of great value to relieve constrictions and incarcerated hernia, to remove fecal matter, mucus and epithelial remnants in chronic enteric catarrh, in dysentery, and in oxyuria, in fact, in all conditions where the system has been depleted or become vitiated by toxic autoinfection, such as hemorrhage. cholera, yellow fever, diabetes, uremia, shock, collapse, etc. In these cases the normal saline solution has been given with the best results, as seen by the improvement in the circulation, the re-establishment of the normal temperature of the body and the stimulating effect upon the nervous system.

Irrigation of the colon has been followed by immediate amelioration of the serious symptoms of the initial stages of appendicitis, and Love ${ }^{2}$ reports several cases in which enemata of one-half gallon of water at $100 \mathrm{~F}$., mixed with soap, were given high up, producing peristalsis and flushing out the irritating matters of undigested food, ptomains, etc.

Shuel ${ }^{3}$ recommends the same treatment in typhoid fever, "to remove at as early date as possible all ptomains and decomposing substances from the colon." The fluid may be readily made to pass the sigmoid flexure and reach to, or near, the cecum. The injection will distend the bowel and remove the accretions from the sacculi. He also cautions against its use during the stage of necrosis of the glandular tissue, as the solitary glands of the large intestine are affected in a large proportion of cases.

Hensell ${ }^{4}$ speaks of the value of colonic irrigations of one-half to one liter, twice a day, reaching up to the ileum, so that their effects are also directly upon the gallbladder and liver; and Löwenthal ${ }^{6}$ reports forty-one cases of catarrhal jaundice cured by this method. These cases were of the type of gastroduodenal catarrh. with symptoms of anorexia, pressure in the gastric and hepatic regions, constipation and great prostration.

\section{AUTHOR'S CASES.}

CASE 1.--Typhoid fever; female, aged 20 years. When first seen, on the eleventh day of the disease, she presented every characteristic symptom, except the diazo-reaction, which was absent throughout. From the fourteenth to the twentyfifth day her evening temperature ranged from 103 to $105 \mathrm{~F}$., which was promptly reduced to 100 by cold spongings. On the evening of the sixteenth day she had a profuse hemorrhage from the bowels, and on the day following several smaller ones. Severe tympanites developed. At this stage irrigation of onehalf gallon of water at $40 \mathrm{~F}$., mixed with soap-suds and onehalf ounce of turpentine, was resorted to, and after this was evacuated, one pint of normal salt solution was injected and allowed to remain. The ice pack was applied to the abdomen. After each irrigation marked improvement took place. The temperature fell several degrees, the pulse, which was from 140 to 150 , and dicrotic, became strong, regular and reduced to 120 to 125 per minute, the nervousness abated, tremor and subsultus tendinum subsided. This treatment was continued until the twenty-fifth day, when defervescence set in and the patient progressed to complete recovery.

CASE 2.-Appendicitis; male, aged 45 years. Patient had just arrived from New York City, where he had a similar attack, which kept him in bed for five days. Having recovered. he attended to his business affairs and then started back to this city, but was seized on the train. When seen he was suffering very acutely, knees drawn up, anxious countenance, clammy sweat, temperature $100 \mathrm{~F}$., pulse 90, exquisite tenderness over McBurney's point and continuous vomiting. Several small doses-1/4 grain-of calomel were given, followed by a Seidlitz powder, ice to abdomen and high irrigation of the bowel with one-half gallon of water and soap at $40 \mathrm{~F}$. After the in testinal tract wats thoroughly cleared out by two more similar injections, at intervals of twelve hours each, the pain ceased, temperature and pulse became normal, and all further symptoms disappeared. The patient was under observation for three months after the attack and has since been in perfect health.

CASE 3.-Appendicitis; male, aged 40 years. Was suddenly taken with violent pain in abdomen, especially pronounced over McBurney's point. Temperature 99.8 F., pulse 90. He presented all the symptoms of Case 2, but was seen several hours earlier in the attack. The same treatment was given and he recovered completely, and has been well since.

In each of the above cases surgical interference was considered; in the first case for impending perforation. in the latter, for the most opportune time to operate, but each improved at the most critical stage, which can only be attributed to the free irrigation of the colon.

B.DLIOGRAPHY. 1089 .

1. Turek, Fenton B.: The Journal A. M. A., 1900, xxxiv, p.

2. Love, I. N.: Ibid., p. 1

3. Shuell, T. J.: N. Y. M. J., 1893, Iviii, p. 255

4. Hensell : Allgemein. Med. Centr. Zeit., Berl., 1896, lxv, p. 897.

5. Löwenthal, Hugo: Berl. Klin. Wochenschr., 1886, xxiii, p. 159. DISCUSSION.

Dr. Danier R. Brower, Chicago, said that too little atten tion has been given by the profession to colonic flushing. He. personally, very much prefers the normal saline solution to soap and water, or anything else which has been proposed for this purpose. Ine solution should be about the temperature of the intestinal tract. Not only does this flushing have a very great effect on the contents of the colon, but there is also a very prompt and remarkably active effect on the renal organs. In cases where the kidneys are deficient, as in certain infectious diseases, a large amount of saline solution thrown into the bowels will very speedily set up action of the kidneys. It is diuretic as well as antiseptic in its relation to the colon, and this feature is often overlooked. He is quite sure that this therapeutic measure is not resorted to as often as it should be.

Dr. O. T. OsBorne, New Haven, Conn., approved of the recommendation of colonic flushing and said that it is not resorted to as often as it should be by surgeons after operations. Some make it a routine practice to inject 1 to 2 quarts of saline solution, but many others do not. The condition of surgical shock is due to paralysis of the vasomotor system, and in that condition the heart gets very weak, because the ventricles are not properly filled. 'The moment the bloodtension goes down, that moment the heart is impaired in its action. Flushing the colon fills the blood-vessels at a time when we know it will take several hours before anything ean be absorbed from the stomach owing to nausea and vomiting, also during this period very concentrated urine is passed. This condition threatens kidney congestion and will be overcome by colonic flushing with normal salt solution.

Dr. J. C. Culber'son, Cincinnati, Ohio, said that as a means of reducing temperature, flushing of the colon with cold water, in infectious diseases, is one of our best remedial expedients. A quart of ordinary ice water will reduce the temperature 1.5 degrees in a case of fever-say 103 F.-if thrown into the rectum and retained there, and it can be repeated as often as thought necessary. It is a very valuable resource, it can do no possible harm and is a source of comfort to the patient.

Dr. M. Berniffim, Philadelphia, said that in the paper read yesterday he had shown, by physiologic experiment, that following an injection of the salt solution there is an antiperistaltic action set up which carries the solution up into the higher part of the small intestine and also into the stomach. Therefore the beneficial action of the large injections of saline solution is more easily understood, as the surface for absorption becomes larger.

DR. G. J. Lochbonuler said that as regards temperature the use of ice water is often very beneficial. but in both of these cases he has used the injection at $40 \mathrm{~F}$, which is sufcient to reduce temperature. He wished to call attention to the fact that the iniection of ice water in the case of hemorrhage reported, with a temperature of $105 \mathrm{~F}$., and about to die, was done as the best method of causing reaction, and he firmly believes that the patient was saved by ehecking the hemorrhace by the stimulating effect of the injection of cold water. But. as he already mentioned, the saline solution given at the body temperature will aid in a great many cases in aborting infection and infectious diseasf. 